\title{
Smart Traffic Congestion Control
}

\author{
Prof. Ranjeet S. Mote ${ }^{1}$, Dharmesh A. Chhajed², Sambhav V. Dwidmuthe ${ }^{3}$, Komal N. More ${ }^{4}$ \\ Professor, Computer Dept., NBNSSOE, Pune, India ${ }^{1}$ \\ Student, Computer Dept., NBNSSOE, Pune, India ${ }^{2,3,4}$
}

\begin{abstract}
India could be a nation that has the one in all the biggest NonLane primarily based road network within the world. Several developed nations these days use Intelligent transit (ITS) to resolve traffic connected issues and supply sleek and safe ride on roads. The road traffic congestions area unit continual drawback in Republic of India. One in all the foremost reasons that cause tie up is that the poor infrastructure and perspective of road users in Republic of India. VANETs area unit wide rising in several developed countries as a more cost-effective, distributive and cooperative tie up system. The well-known tie up drawback in each surroundings has negative impact on several areas together with economy, surroundings, health and life-style. Recently, variety of solutions supported vehicle -to-vehicle communications were planned for tie up detection and management. Vehicular Ad hoc networks (VANETs) area unit accustomed collect and combination period of time speed and position info on individual vehicles to optimize signal management at traffic intersections. Vehicular Ad hoc networks (VANET) could be a promising technology to alter the communications among vehicles.
\end{abstract}

Keywords: Traffic Congestion, Vehicular Ad Hoc Networks (VANET), Traffic Information System.

\section{INTRODUCTION}

During the last decades, worldwide road traffic density has been increasing year when year. This case has diode to the very fact that, currently, expressway traffic congestions square measure one amongst the foremost common phenomena that motorists need to face in their journeys. excluding being a quite-stressful expertise for current drivers, they even have a negative impact on the surroundings and also the economy. additionally, the road traffic conditions have an effect on the protection of the population since one. 2 million individuals worldwide square measure calculable to be killed annually on the roads. For this reason, these days the automotive trade and governments invest several resources to extend road safety and traffic potency, additionally on cut back the impact of transportation on the surroundings. one amongst the foremost promising areas of analysis is that the study of the communications among vehicles and artificial language ad-side units, or a lot of specifically the Vehicular Ad-hoc Networks (VANETs). this sort of networks square measure self-configuring networks composed of a group of vehicles And components of edge infrastructure connected with one another while not requiring an underlying infrastructure, causing and receiving data and warnings regarding this traffic state of affairs.

\section{RELATED WORK}

M. Sheela Devi and K. Malar proposed a D-FPAV(Dynamic Fair Transmit Power Adjustment) [1]Algorithm that support both traffic \& non traffic situation, in the algorithm to calculates transmit power control value at each vehicle with the help of beacon message information. Then interchange the transmit power value calculated among the neighbouring vehicle and last select best power value .

R. Yugapriya, P. Dhivya, M. M Dhivya, and Mr. S. Kirubakaran aver proposed method to reduce traffic signal control problem to the problem of scheduling jobs on processor, and propose algorithm called the Greedy Forwarding Algorithm [2] to transfer the data faster. The information consists of Speed data which can be gathered from the vehicle speedometers, and position information data can be gathered using GPS receivers fitted to the vehicles.

My Driss LAANAOUI, and Pr. Said RAGHA Y to solve the broadcast storm problem using greedy forwarding and decomposition zones, in which firstly used GyTAR (Improved Greedy Traffic Aware Routing protocol)[3] which is an intersection-based geographical routing protocol capable to find robust routes within city environments. It consists of two modules: (i) Selection of the junctions through which a packet must pass to reach its destination, and (ii) an improved greedy forwarding mechanism between two junctions. Hence, using GyTAR, a packet moved successively closer towards the destination along streets where there are enough vehicles to provide connectivity.

Milos Milosevic and Vaseline Rakocevic present an algorithm designed to enable each vehicle in the network to detect and quantify the level of traffic congestion in completely distributed way, independent of any supporting infrastructure and additional information such as traffic data from local authorities [4]. Therefore divided this algorithm in two mechanisms: - (i) congestion detection and quantification, and (ii) information dissemination [4]. 
Cheng-Wei Fan, Ke-Chian Su, Hei-Min Wu, Wei-Lin Chang and Yao-Hsin Chou present, position base broadcast module, named Broadcast Control Unit (BC Unit), in order to reduce the re -broadcast nodes and minimize emergent message conflict. If quantity of vehicles increased, the message delivery rate will decrease significantly due to the message contentions and collisions.

\section{IMPLEMENTATION}

The Traffic App is installed in the driver's smart phone and used to obtain traffic information. TheTraffic App has been developed in the Android platform. Once this app is initialized, it enables the location of the smart phone. The entire traffic congestion management systemconsists of two modules: (ii) Congestion Detection Module and (iii)Congestion Dissemination Module. The Traffic App installed in the driver's smart phone. The remote serverconsists of a MySQL database.Home page will show user login, map, history and notification as shown in figure 1.

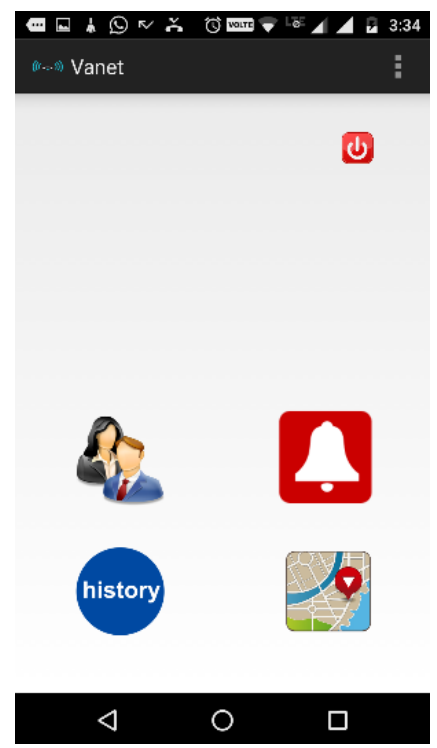

Figure 1.

User will login to app by entering login credentials such as login id and password. If user is not registered then he/she will registered as new user as shown in figure 2(a). User will enter route by entering details. User will enter source and destination as shown in fig 2 (b). As shown in figure 3. Map will be shown showing route of travelling. Result of traffic level will be shown as low, medium and high.

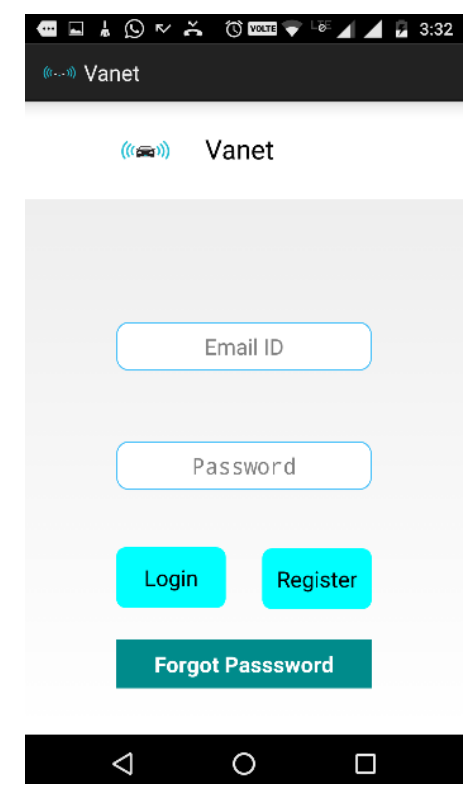

Figure 2(a) 
International Journal of Advanced Research in Computer and Communication Engineering ISO 3297:2007 Certified

Vol. 6, Issue 5, May 2017

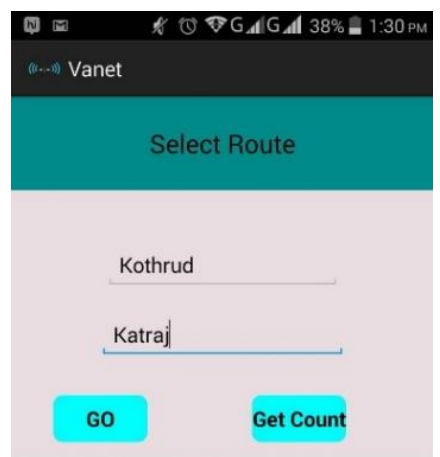

Traffic level :: Low

Figure 2(b)

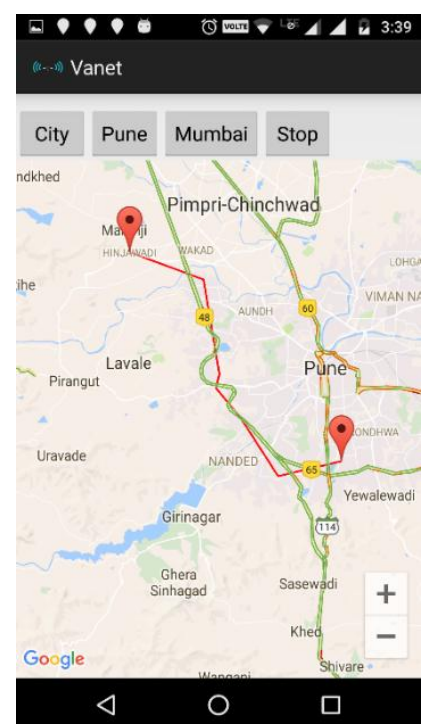

Figure 3

Route enter by user is given on map. Traffic congestion is shown where ever user have entered quantity as shown in figure 3

Finally user will get traffic notification on their mobile, showing message "you are in range of traffic" as shown in figure 4

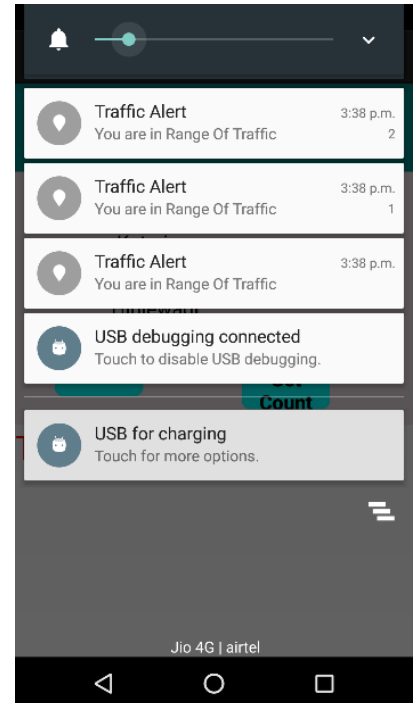

Figure 4 


\section{RESULT AND ANALYSIS}

In this way, we have implemented our application. This application is user friendly. It will help to maintain traffic control system. User will come to know about traffic situation. By knowing traffic situation system, traffic control system is managed.

\section{REFERENCES}

[1] Lecture notes in Traffic Engineering And Management by Civil department, IITB.

[2] Robinson, R. "Problems in the urban environment: traffic congestion and its effects."Wollongong Studies in Geography (1984): 14.

[3] Mittal, Narendra Mohan, and SavitaChoudhary. "Comparative Study of Simulators for Vehicular Ad-hoc Networks (VANETs)." InternationalJournal of Emerging Technology and AdvancedEngineering Website: www. ijetae. com (ISSN 2250-2459, ISO 9001: 2008 Certified Journal, Volume 4,Issue 4(2014).

[4] Zeadally, Sherali, Ray Hunt, Yuh-Shyan Chen, Angela Irwin, and Aamir Hassan. "Vehicular adhoc networks (VANETS): status, results, and challenges." Telecommunication Systems 50, no. 4 (2012): 217-241.

[5] El-Sersy, Heba, and Ayman El-Sayed. "Survey of Traffic Congestion Detection using VANET."

[6] Zeadally, S., Hunt, R., Chen, Y. S., Irwin, A., \& Hassan, A. (2012). Vehicular ad hoc networks (VANETS): status, results, and challenges.Telecommunication Systems, 50(4), 217241.

[7] Teixeira, Fernando A., et al. "Vehicular networks using the IEEE 802.11 p standard: an experimental analysis." Vehicular Communications 1.2 (2014): 91-96.

[8]http://www.gpsinformation.org/dale/nmea.htm\#G GA[Data accessed on 13/12/2014].

[9] Kausar, Tanwee, Priyanka Gupta, Deepesh Arora, and Rishabh Kumar. "A VANET based Cooperative Collision Avoidance System for a 4-Lane Highway."

[10] Akhtar, Naheed, SinemColeriErgen, and OznurOzkasap. "Vehicle mobility and communication channel models for realistic and efficient highway VANET simulation." Vehicular Technology, IEEETransactions on 64.1 (2015): 248-262. 